\section{Introduction to Mass Spectrometry An ACS Video Course}

The American Chemical Society, Washington, DC 20037 (c) $1993, \$ 995$

Reviewed by O. David Sparkman

Mass Spectrometry Consultant

4045 Eagle Ridge Drive

Antioch, CA 94509

This ACS-produced video course on mass spectrometry consists of two separate tapes (Instrumentation for Mass Spectrometry, $20 \mathrm{~min}$, and Interpretation of Mass Spectra, $60 \mathrm{~min}$ ) and a 125-page study guide. J. Throck Watson was the technical advisor on this project and authored the study guide. The course presents many principles presented in his 1985 book Introduction to Mass Spectrometry (Raven Press).

The presentations in both videos are very dynamic. Through the use of outstanding narration, extremely well-done animation, and actual clips of a mass spectrometer in use, the concepts of sample introduction through a gas chromatograph and solid probe and electron and chemical ionization are well described. There are no dead moments where nonmeaningful events are shown without narration. This will keep the viewer's interest all the way through both videos.

The Instrumentation for Mass Spectrometry video will give the viewer a good understanding of classical mass spectrometry and how quadrupole, magnetic sector, and double-focusing mass spectrometers work. This video also makes extensive use of some of the best animation I have seen in the description of how things work in a mass spectrometer. This will allow those who want an understanding of mass spectrometry to achieve this through a true course of self-study.

The Interpretation of Mass Spectra video is inappropriately titled. This six-part video actually does an excellent job of showing how to deal with mass spectral data. Although the general principles of interpretation of electron ionization mass spectra are very well presented, emphasis is placed on how to select which spectrum to interpret. The problem sets in the guide help to emphasize the "Introduction" part of the title. They deal with understanding the whole mass spectrum and the data from which the mass spectrum comes, rather than concentrating on the meaning of individual peaks as they relate to one another. The study guide contains a very well presented "General Strategy for Interpreting Mass Spectra" that will serve those who use this course for a long time in their future study of mass spectrometry. The nitrogen rule, use of isotope ratios to determine elemental composition, heterolytic and homolytic fission, intermolecular rearrangements, the rings plus double bond rule, and reasonable and unreasonable losses used in this strategy are well presented in the video and study guide.

Although the six sections in the Interpretation of Mass Spectra video are labeled, better prompting of the viewer to stop the tape and use the study guide should have been implemented. There is nothing in the video that tells the viewer it is time to refer to the study guide and work a problem set. There is no documentation, except for an ambiguous statement embedded in the "A Word to the User" page of the study guide, that explains the use of the guide in conjunction with the tapes.

Viewers of the section on background subtraction in gas chromatography/mass spectrometry data may be misled by the location of the appropriate background scan relative to the sample scan. Although this is well presented in the study guide, the background scan in the video is taken on the front side of the peak where the amount of column bleed is less. A glaring deficiency in the presented material is the lack of information on library searching and the use of large commercially available mass spectral databases in electron ionization mass spectrometry. For completeness, this would have to be added in any course of study that uses these materials.

The total amount of time required for this course, which includes reviewing the study guide, working the seven problem sets, and reviewing your answers with those in the "Answer to Problems" section is between 10 and 20 hours, depending on the degree of understanding you want to obtain. This ACS course is ideal for use by new mass spectrometry operators in industry and students in an instrumental analysis course. This video course can be augmented with a workbook of spectra that require interpretation and hands-on laboratory experiments to broaden the learning experience.

The ACS Introduction to Mass Spectrometry is an excellent addition to any mass spectrometry educational library and will serve users for many years. 